Marie VOŽDOVÁ

Université Palacký d'Olomouc

\title{
LA RÉCEPTION DU THÉÂTRE DE JEAN GIRAUDOUX EN TCHÉCOSLOVAQUIE DANS L'ENTRE-DEUX-GUERRES
}

L'objectif de cette étude est de présenter la réception des drames giralduciens en Tchécoslovaquie dans la période interbellique, à un moment où la culture française occupait une place exceptionnelle, place qu'elle a perdue après 1945 et n'a plus jamais retrouvée ensuite. La présente étude s'appuie sur les résultats des travaux de recherche effectués grâce au projet scientifique subventionné par l'État et portant sur la réception de la production dramatique française en Moravie. ${ }^{1}$ Comme source d'information, nous utiliserons les articles de critiques dramatiques publiés dans les journaux tchèques de l'époque tels que Lidové noviny, Venkov, Národní listy, Rudé právo, Právo lidu, Lumír, Národní a Stavovské divadlo, Československé divadlo, Národní divadlo, Divadelní noviny, Divadelní zápisník, Otázky divadla a filmu, Kulturní politika, Rozpravy Aventina et Prager presse, qui se situent entre avril 1929 et avril 1938. Même si les sources de la presse ne sont pas disponibles dans leur totalité (les dépositaires et les archives de coupures de journaux des théâtres tchèques n'ont pas été épargnés de catastrophes, tant sociales que naturelles ${ }^{2}$ ), nous avons pu en consulter une centaine, et ainsi nous faire une image assez objective de l'atmosphère qui accompagnait les premières des pièces giralduciennes.

Dans la période de l'entre deux-guerres on a joué, en Tchécoslovaquie, cinq drames de Jean Giraudoux : Siegfried (1929), Amphitryon 38 (1931), Intermezzo (1934), La guerre de Troie n'aura pas lieu (1936) et Electre (1938). ${ }^{3}$ Toutes les pièces mentionnées ont été présentées sur la scène du Théâtre National à Prague, dans la capitale tchèque, sauf Siegfried, qui a été également joué par la troupe du Théâtre moravosilésien à Ostrava, c'est-à-dire dans un petit théâtre régional, et La guerre de Troie n'aura pas lieu qui a été encore étudiée à Ostrava et à Brno, soit deux autres scènes régionales. Siegfried est la première pièce de Giraudoux à avoir été introduite sur les scènes théâtrales tchèques tandis que La guerre de Troie n'aura pas lieu est considérée comme la plus réussie.

Siegfried a été présenté pour la première fois au Théâtre National de Prague le 24 avril 1929, par l'excellent metteur en scène Vojta Novák, une année après sa première représentation à la Comédie des Champs-Elysées à Paris. ${ }^{4} \mathrm{~A}$ la

\footnotetext{
${ }^{1}$ Le projet est réalisé par le Département de Langues et Littératures Romanes à la Faculté des Lettres de l'Université Palacký d'Olomouc grâce au soutien financier de GAČR, numéro de projet 405/05/2725, Les drames français et italiens sur les scènes moraves.

${ }^{2}$ Cf. ŠRÁMEK, Jiř́ : «Cyrano de Bergerac d'Edmond Rostand sur les scènes moraves », Studia romanistica 6, Ostrava, p. 194.

${ }^{3}$ La représentation planiphiée de Cantiques des cantiques en 1938 n'a pas été réalisée.

${ }^{4}$ Siegfried a été représenté pour la première fois à la Comédie des Champs-Elysées le 3 mai 1928 avec la mise en scène de Louis Jouvet. Le texte de la pièce a été traduit en tchèque par Růžena Thonová. Pour la représentation de Prague : scène V. Hofman, acteurs V. Vydra, L. Dostalová, E. 
représentation tchèque a précédé l'article du correspondant parisien Richard Weiner du journal Lidové noviny qui y a exprimé sa grande déception par Siegfried-drame en comparaison avec le roman. La pièce est incomparable avec le roman, plein de tirades, qui deviennent dans le drame le balaste. Siegfried était d'après lui destiné pour une adaptation scénique moins que toute autre chose de Giraudoux. (WEINER, 1928 : 7) Néanmoins le public et la critique tchèques ont accueilli favorablement ce drame sur le personnage de l'écrivain français Jacques Forestier qui a perdu la mémoire et qui, sous le nom de Siegfried von Kleist, est devenu le chef de l'Allemagne pour enfin, grâce à l'amour de Geneviève, revenir à son pays d'origine. Hanuš Jelínek, écrivain et traducteur tchèque, soulignait, qu'il était très difficile de traduire une œuvre qui exprime l'esprit de la race et qu'il est peut-être même impossible qu'un acteur étranger sache mesurer la dose de poésie, de moquerie, d'émotion, d'ironie et de tendresse qui sont parties intégrantes de ses personnages. «L'ami Giraudoux est certainement l'un des esprits les plus européens de la France d'aujourd'hui, il n'a ni l'ombre du nationalisme limité ou de la haine, et malgré cela reste, avec Colette, le plus français de tous les auteurs français d'aujourd'hui. Dans chacun de ses personnages il y a quelque chose de l'indéfini, qui n'appartient qu'aux Français, chacune de ses phrases même si elle a un sens cosmopolite, prend sa source dans la pure tradition française », écrivait-il. (JELÍNEK, 1928/1929: 429) ${ }^{5}$

Pour la critique tchèque l'explication du drame est double, l'œuvre symbolise deux races, germanique et romane, et est une sorte d'appel pour une dépolitisation de la vie, mais aussi un résumé de la guerre - car l'Allemagne, après la guerre, a été reconstruite aussi par les Français. (-JP-, 1928: 7) La critique dramatique tchèque comprend Siegfried comme un drame des frontières, réelles mais aussi abstraites dans l'intérieur de chacun, limites de son moi, de sa relation à la patrie, et de ses préjugés. Après la représentation à Ostrava, la critique régionale a constaté que la pièce «passionne par chaque mot » et surtout par de l'esprit ce qui «à nos yeux caractérise les Français ». Siegfried était d'après elle, dès le commencement un doux héros, un homme plutôt français qu'allemand et très malléable qui se décide d'après la voix de son cœur. (BÁR, 1929/1930 : 119) Il représentait pour nos critiques l'incarnation la plus parfaite de l'Européen. Le sujet giralducien allait bien avec le quasi mythe que s'étaient créés les Tchèques pendant des siècles, celui de la peur éternelle de l'Allemagne et avec lui, la recherche et l'espoir d'une protection de la part de la France.

Les critiques tchèques ont apprécié aussi le renouvellement giralducien dans la forme du théâtre moderne, ce qui semblait être aussi pour eux une preuve symbolique de la victoire de la France sur Allemagne : «La victoire est double la première dans la pièce, la seconde par la pièce en tant qu'œuvre du théâtre

\footnotetext{
Vrchlická, K. Dostal, L. Veverka. Pour la représentation d'Ostrava en octobre 1929 : mise en scène Skála, scène Vl. Kristin, acteurs Skála, J. Svobodová, T. Hodanová, V. Leraus.

${ }^{5}$ En se souvenant de l'article de Junia Letty, un écrivain franco-belge qui, dans la revue Europe centrale, avait reproché au Théâtre National la vitesse de leurs études des pièces françaises et le petit nombre de répétitions, Hanuš Jelínek rappelle la caricature pragoise de Topaz de Pagnol et son échec, et il se demande si le théâtre achète en même temps que le droit de production de la pièce le droit de la changer, de la détruire et de la saboter. Il repproche aux réalisateurs qu'ils ont « ajouté la dernière phrase pour améliorer le texte de monsieur Giraudoux. » Jelínek, Hanuš, « Théâtre », Lumír, Prague, tome 55, 1928/1929, № 9, pp. 429-431.
} 
moderne - il suffit de se souvenir comment, ces dernières années, une recherche furieuse d'une forme nouvelle a dominé le théâtre allemand. » (B., 1929 : 7).

Quant aux résonances négatives, on a reproché à Giraudoux d'affaiblir le côté érotique des deux femmes entre lesquelles se trouve le héros, comme dit le critique tchèque «un côté si typique des Français autrement », et aux acteurs tchèques de parler beaucoup, la pièce souffre ainsi d'une grandiloquence et d'une abondance de mots d'esprit. (-JP-, 1929: 132) De même, le rédacteur Paulík de la revue Rozpravy Aventina est contre l'application de la méthode prosaïque giralducienne au drame, qui ne marche pas du tout, et il souligne que les acteurs tchèques ont encore accentué ces fautes de l'auteur. La mise en scène était d'après lui trop monumentale et elle n'a pas su effectuer le vrai mélange des images réelles et irréelles qui n'étaient que juxtaposées. (PAULÍK, 1928/1929 : 332)

La deuxième pièce giralducienne, Amphitryon 38, a été jouée à Prague le 14 mars 1931, un an et demi après sa représentation parisienne, dans une mise en scène d'un artiste primordial, Karel Hugo Hilar, sous le titre du Nouvel Amphitryon (Nový Amfitryon). ${ }^{6}$ Ainsi le traducteur et le metteur en scène ont préféré l'omission du chiffre dans le titre et par là ont déjà un peu ignoré l'intention de l'auteur. Richard Weiner, le correspondant parisien de Lidové noviny, dès qu'il apprend qu'on va jouer Amphitryon à Prague, ne tarde pas de nouveau à rendre sa critique : «Vous, heureux Tchécoslovaques, qui savez déjà ce que signifie le chiffre 38 dans le titre, ce que le public à Paris ne savait pas du tout [...] On peut se dire que le dramaturge qui se jette sur le sujet, avant lui travaillé déjà trente-sept fois, entre autres par Molière, [...] sera certainement un peu trop plein d'esprit. Et on a raison, Amphitryon ne sert que de prétexte pour des jeux de mots, aphorismes etc. Monsieur Giraudoux a rempli un vide sensiblement manquant : on se plaignait souvent que les salons de conversation n'existent plus, mais l'auteur en a installé un sur la scène de la Comédie des Champs Elysées. Si le vieux Boileau vivait, il aurait écrit son hémistiche célèbre différemment : le droit d'avoir de l'esprit, on se le procure en payant. » (WEINER, 1929 : 9)

La représentation pragoise a montré une œuvre de pure poésie. Hilar avait excellé comme toujours dans la division de l'espace scénique : à gauche deux colonnes antiques pour les entrées des dieux, à droite l'escalier pour Sosie et au centre l'intérieur de la maison d'Amphitryon caché de temps en temps par le rideau et au-dessus de tout la mer. (PÍŠ́A, 1931 : page illisible)

Mais cette fois-ci, malheureusement, le correspondant Weiner avait un peu raison. Le public et la critique tchèques ont automatiquement comparé Giraudoux à Molière qui régnait en tant que dramaturge français en Tchécoslovaquie. Giraudoux ne possédant pas le comique spontané de Molière, a été jugé trop froid, trop artistique. "Tout autre Français serait plus gai que Giraudoux », écrivaient les critiques tchèques. Les attentes du public ont été déçues, on riait beaucoup moins et «l'atmosphère dans la salle était un peu sèche comme l'est aussi la pièce. » L'œuvre, d'après les Tchèques pourrait être plus vivante et «plus gamine à la

\footnotetext{
${ }^{6}$ Amphitryon 38 a été représenté pour la première fois à la Comédie des Champs-Elysées le 8 novembre 1929 avec la mise en scène de Louis Jouvet. Le texte de la pièce a été traduit en tchèque par Růžena Thunová. Pour la représentation tchèque : scène V. Hofman, acteurs O. Scheinpflugová, B. Karen, E. Kouhout, R. Nasková, J. Kronbauerová, J. Steimar.
} 
française. » «Où est restée l'ironie giralducienne ?» se demandaient-ils. La critique a même reproché à la dramaturgie du Théâtre National le fait de choisir dans le répertoire cette pièce qui a eu du succès en Allemagne comme si cela devrait être pareil chez nous. Des questions du type « Le Théâtre national, ne veutil pas aussi par hasard créer une union théâtrale avec l'Allemagne ? (IBID.) ont laissé voir l'atmosphère anti-allemande dans certains rangs de l'élite culturelle.

Otokar Fischer dans Lidové noviny apprécie la nouveauté giralducienne dans la conception du personnage féminin d'Alcmène qui devient le personnage central de la pièce. Mais d'après lui, la pièce manque de réalisme et l'envoi moral. Au dialogue final entre Alcmène et Jupiter il reproche trop de dialectique, toute la pièce n'est faite que d'apparences et de jeu qu' « on ne peut imaginer que difficilement» et que «nous ne pouvons pas transmettre dans notre vie citoyenne. » (FISCHER, $1931: 7$ )

Dans les années trente, les spectateurs tchèques apprennent par les journaux que les théâtres parisiens sont tombés dans un embarras de répertoire et qu'à part d'anciennes pièces renouvelées, il n'y a que l'œuvre de Jean Giraudoux qui mérite d'être mise en scène. C'est pourquoi on attend avec impatience sa nouvelle pièce. Intermezzo est joué en Tchécoslovaquie au Théâtre National sur la scène de Stavovské divadlo sous le titre de Isabelle au carrefour (Isabela na rozcestí) le 9 janvier 1934, dans une mise en scène de Jiří Frejka, avec une excellente traduction de Jindřich Hořejší (qui par cet autre titre a mis l'accent sur les crises d'adolescence de l'héroïne) et avec la musique de Podaševský qui est en harmonie absolue avec les dialogues et la danse. ${ }^{7}$

Giraudoux est salué par la critique en tant que l'un des derniers grands poètes sur la scène mondiale dont la force tient dans un dialogue bien construit. Elle chante une atmosphère poétique brumeuse pleine de jeux d'ombre et de lumière et des pensées cachées. D'après Josef Träger de Právo lidu, Giraudoux, par le biais du destin d'Isabelle, dévoile le mystère du changement de la fille en femme. Il s'approche de la foi primitive du peuple qui croit en la liaison des filles avec des spectres d'entre la vie et la mort. Le caractère d'Isabelle reflète ainsi d'après lui l'ironie amère de l'auteur qui dans la femme trouve la capacité de désabusement des rêves d'adolescence tandis que les hommes conservent toute leur vie la tentation de l'aventure d'imagination. Träger est persuadé qu'au Théâtre National à Prague, dans la mise en scène de monsieur Jiř́ Frejka, cette œuvre giralducienne avait trouvé sa forme scénique parfaite. Le metteur en scène tchèque a d'après lui «dématérialisé la pièce, ce qui était tout à fait en accord avec l'intention de l'auteur. On a seulement regretté que dans le dernier acte de la pièce, l'auteur dénoue trop vite l'histoire qu'il avait si lentement et si soigneusement mis en collision. »: (TRÄGER, 1934 : page illisible)

Hanuš Jelínek dans Lumír a très bien exprimé les opinions contradictoires de la critique et du public. Il a apprécié la valeur littéraire et dramatique de cette comédie en trois actes, mais constaté que le public est resté embarrassé devant une

\footnotetext{
${ }^{7}$ Intermezzo a été représenté pour la première fois au Théâtre Louis Jouvet (Comédie des champsElysées) le $1^{\mathrm{er}}$ mars 1933 dans la mise en scène de Louis Jouvet avec la musique de Francis Poulenc. Le texte de la pièce a été traduit par Jindřich Hořejší. Pour la représentation tchèque : scène $B$. Feuerstein, acteurs O. Scheinpflugová, E. Kohout, F. Roland, Pešek, Průcha, Boháč.
} 
pièce faite du mélange fragile des rêves, des symboles, de la réalité et de la satire. Même un metteur en scène aussi doué que Frejka n'a pas su, d'après Jelínek, trouver la juste mesure entre les scènes réelles et irréelles. «Jean Giraudoux, explique-t-il, est un si pur produit de la tradition et du milieu français qu'il est vraiment difficile de demander au public médiocrement instruit de le comprendre complètement. » (JELÍNEK, 1933/1934 : 172) Aussi František Götz dans le journal Národní divadlo regrette que la poésie de Giraudoux n'enrichisse qu'un spectateur attentif et penseur. (GÖTZ, 1933/1934 : 3) De l'incompréhension totale d'une partie du public témoigne la réaction fâchée du Josef Träger qui lui reproche d'ignorer les lois les plus simples du comportement en société et de gêner les autres de ses toux, aspersions et éternuements comme manifestation de l'indignation sur la pièce qu'on ne comprend point et qui exige plus que d'être consommée comme une farce ordinaire. «Mais la réaction de cette partie ignorante du public », dit-il, « ne peut que faire honneur à Giraudoux. » (TRÄGER, 1934 : page illisible)

La plus connue mondialement et la plus jouée des pièces giralduciennes, $L a$ guerre de Troie n'aura pas lieu (Trojská válka nebude), a été successivement étudiée la même année par les théâtres de la ville de Prague et jouée sur la scène de Komorní divadlo, puis en Moravie du sud au Zemské divadlo de Brno et en Moravie du nord au Théâtre National moravosilésien d'Ostrava. ${ }^{8}$ La première représentation pragoise a eu lieu le 29 octobre 1936, soit presque une année après la première parisienne. Gustav Winter, le correspondant parisien de Právo lidu, s'exalte après la représentation parisienne : «Aucune traduction ne peut reproduire la langue extraordinairement belle, dans laquelle est écrit l'original. Et je doute qu'on puisse trouver un acteur qui saurait présenter Hercule comme Louis Jouvet, plein d'énérgie et de douleur à la fois, et certainement on ne trouvera nulle part une actrice qui puisse évoquer Hélène aussi bien que Madeleine Ozeray, belle, blonde, l'incarnation du péché innocent!» (WINTER, 1935: 6) Néanmoins la traduction tchèque élégante de Jindřich Hořejší et la mise en scène de František Salzer ont apporté un grand succès. L'esprit et la spiritualité de la pièce ont été également bien traduits par l'interprétation tchèque et le public l'a regardée avec une compassion rarissime. La réalisation scénique intéressante sur ses espaces bleu-blancs et à l'escalier blanc a augmenté l'atmosphère symbolique des scènes contre la guerre. Si dorénavant les pièces de Giraudoux pourraient sembler ne s'adresser qu'à un public choisi, La guerre de Troie n'aura pas lieu a trouvé sa voie vers le large public.

Les critiques tchèques ont constaté que la pièce était écrite avec fraîcheur et qu'elle était pleine de l'esprit français que ne peut comprendre dans tous ses détails qu'un Français. Sous une forme bien allégorique, elle reflète d'après eux l'opinion de l'auteur que la guerre était et sera toujours. (PETR, $1936: 166$ ) «Je connais la divagation ironique théâtrale du dernier héritier français de la préciosité

\footnotetext{
${ }^{8}$ La guerre de Troie n'aura pas lieu a été représentée pour la première fois au théâtre de l'Athénée le 21 novembre 1935, sous la direction de Louis Jouvet. Le texte de la pièce a été traduit en tchèque par Jindřich Hořejší. Pour la représentation de Prague : acteurs Brožová, Glázrová, Bušová, Korbelář, Vnouček, Gollová, Skorkovská. Pour la représentation de Brno : mise en scène Skřivan, scène Fr. Muzika, acteurs Nedbal, Švabíková, Lokša, Hoger, Lukeš, Pavlíková.
} 
déjà de la représentation parisienne, mais l'entendre dans une réalisation presque statique comme des dialogues costumés, ce fut pour moi un grand plaisir mélancolique, » écrivait Hanuš Jelínek dans Lumír (JELÍNEK, 1936/1937 : 119). On a trouvé immédiatement l'allusion allégorique de la pièce à la situation historique, la guerre est alors vraiment devenue le destin des gens, même si le héros courageux fait tout pour l'arrêter. «Le poète Demokos rappelle des poètes patriotes qui parlent de l'honneur national. Mais les poètes patriotes à l'arrière sont toujours plus énergiques que les combattants au front », dit le redacteur de Právo lidu et il ajoute, que chez Giraudoux, c'est le poète qui provoque la guerre, comme « aujourd'hui c'est le cas du journaliste avide de sensation ». (WINTER, $1935: 6)^{9}$ Le succès retentissant de la pièce était donc dû au choix d'un thème d'actualité qui touchait tous les spectateurs tchèques.

Le 11 avril 1938, un an après sa première représentation parisienne, a été jouée au Théâtre National Electre de Giraudoux sous le titre de la Nouvelle Electre (Nová Elektra) dans une mise en scène de Karel Dostal. ${ }^{10}$ La pièce a eu un succès immense, le public a vivement applaudi, pour les théâtres pragois c'était l'événement de la saison, la plus travaillée et la plus réussie des représentations de l'année. «Belle, rare, soirée réussie ! ${ }^{11}$ ont écrit les critiques enthousiasmés. «Quelle danse de l'ours montre le dramaturge américain [allusion à O'Neill, joué avant] en comparaison avec le Français, qui dans la Nouvelle Electre surmonte vraiment les lois de la pesanteur. La représentation d'Electre est l'orgueil de notre cycle des soirées du théâtre ! ${ }^{12}$ Ce grand succès est certainement dû aussi à Olga Scheinpflugová, actrice très célèbre de grand talent de l'entre-deux guerres qui jouait le rôle d'Electre. Son Electre était pleine de haine, hallucinée par la pensée de la vengeance ce qui l'a privée de tout sentiment humain et a ajouté une grandeur glaciale. La musique scénique suggestive de Miroslav Ponc ainsi que la traduction de Jindřich Hořejší caractéristique par un soin de la parole claire et pure, ont encore augmenté l'effet final de la pièce. Hanuš Jelínek avoue que pour lui les représentations tchèques des pièces françaises vues avant à Paris sont presque toujours une déception. Mais Electre de Giraudoux peut d'après lui être bien comparée à sa représentation parisienne et même à plusieurs endroits elle la domine. (JELÍNEK, 1937/1938: 326) La représentation tchèque a intentionnellement pris de la distance avec celle de Paris, on a fait plusieurs raccourcis de texte, omis l'intermezzo avec le jardinier et on a ajouté de la majesté au personnage du mendiant.

La critique tchèque s'est tout de suite mise à expliquer la pièce et à chercher de quel côté était la sympathie de l'auteur dans ce double conflit entre les intérêts personnel et commun, ceux de l'individu et de l'Etat, et a constaté que le

\footnotetext{
${ }^{9}$ Il est intéressant que le théâtre allemand à Prague ait par hasard donné en même temps la même pièce, mais dans sa version raccourcie. L'œuvre avait ainsi perdu des passages intéressants et fort beaux. Les scènes lyriques étant très réduites, la version allemande rappelait la farce pacifique.

${ }^{10}$ Electre a été représentée pour la première fois au théâtre Louis-Jouvet (Athénée) le 13 mai 1937 sous la direction de Louis Jouvet. Le texte de la pièce a été traduit en tchèque par Jindřich Hořejší. Pour la représentation tchèque : scène Fr. Muzika, acteurs O. Scheinpflugová, M. Pačová, B. Karen, J. Dohnal, F. Smolík, J. Pivec,V.Vydra, J. Kronbauerová, musique M. Ponc.

${ }^{11}$ Cf. Engelmüller, Karel de Národní politika, cité d'après Národní divadlo, le 30 mars 1938, p. 11.

${ }^{12}$ Cf. A.V. de Pražské noviny, cité d'après Národní divadlo, le 30 mars 1938, p. 11.
} 
dramaturge ne fait que de montrer le conflit tragique de ces deux principes dont chacun porte en soi un morceau de vérité. A. M. Píša de Právo lidu trouve que « le spectateur tchèque, nourri par les pensées de Masaryk, connaît une solution qui suppose l'équilibre de deux côtés opposants de ce conflit: la protection de la patrie, de la nation et de l'Etat prend sa source dans l'idée morale de vérité. Il s'agit d'une lutte temporelle au nom de valeurs éternelles, saintes devant l'éternité. La vérité terrible ainsi ne tue pas la nation mais au contraire elle la nourrit et la fortifie. » (Ṕ́̌́s, 1938 : 4)

Hanuš Jelínek a admiré la simplification et la dépathétisation avec lesquelles Giraudoux a travaillé le mythe antique en lui ajoutant un nouveau sens ironique. Il comprend Electre comme un cri passionnant du désir de justice absolue même s'il signifie la mort et la destruction de toute la ville et de tout le pays. (JELÍNEK, 1937/1938 : 326) A. M. Brousil trouve que le motif central d'Electre, c'est-à-dire qu'elle ne veut pas accepter la patrie chargée du crime et que la patrie lui est une véritable patrie seulement dans la vérité pure, est un motif incroyablement slave. Aussi le fanatisme d'Electre est d'après lui très peu français. Il ressent le manque de dynamisme et le caractère trop statique et plutôt conversationnel de la pièce. (BROUSIL, 1938: 6) La seule opinion tout à fait négative dans tout le champ littéraire est apportée par le critique communiste Julius Fučík dans Rudé právo, le journal d'extrême gauche. Cette réaction négative très féroce reflète la haine révolutionnaire de l'auteur qui l'empêche de voir le contenu de la pièce. ${ }^{13}$

Pour conclure, nous pouvons constater que les drames de Jean Giraudoux ont attiré l'attention dans la Tchécoslovaquie de l'entre-deux-guerres. Les spectateurs tchèques se sont vivement intéressés à l'œuvre de Jean Giraudoux, dont chacune des pièces écrites à cette époque-là a été attentivement étudiée par l'ensemble du Théâtre National de Prague et présentée au public au plus tard un an après sa représentation à Paris. Les critiques dramatiques tchèques ont toujours été bien informés des représentations parisiennes, certains d'entre eux les ont même vues et ont pu comparer les versions françaises et tchèques, leurs spécificités de mise en scène ainsi que la pertinence de leurs traductions en tchèque. ${ }^{14}$ Le théâtre

13 «Ces derniers temps, le Théâtre National nous présente le luxe, c'est-à-dire ce qui ne devrait pas exister », proclame-t-il. «Et il faut combattre le luxe dans l'art. Cocteau, l'homme des effets faciles, fut le premier de ce qui est vraiment vain. Jean Giraudoux avec sa vieille Electre en est maintenant le deuxième. Son Electre est absolument chaotique. Le chaos n'est pas une ouvre et la Nouvelle Electre est un chaos misérable. La construction de la pièce est acceptable, mais l'auteur ne sait pas ce qu'il veut. Electre doit incarner la justice révolutionnaire. Mais Giraudoux ne sait pas imaginer un révolutionnaire. Un Révolutionnaire sans amour du peuple n'est pas un révolutionnaire. C'est pourquoi Electre te dégoûte comme une créature hystérique, elle te dégoûte comme quelque chose de mauvais et en comparaison avec elle tu trouves même que le sale meurtrier Aigiste est sympathique. Et ça non seulement toi, spectateur, mais aussi l'auteur lui-même qui est resté perplexe devant le texte qu'il a écrit. Alors encore une fois nous avons ici un grave problème actuel qui est intellectuellement surcalculé jusqu'à atteindre le point de congélation. C'est froid. Et combien de mots sonores il a fallu dépenser pour une traduction parfaite de cette pièce chaotique, quels efforts ont fournis les acteurs pour leurs rôles, mais tout ça n'a pas abouti à une nuit de poètes ! Il n'y a que la scène qui avait son ordre, inébranlable par le chaos des mots giralduciens. » (Fučík, 1938 : sans pagination)

${ }^{14}$ Il faut souligner que les textes de Giraudoux ont été préparés par les meilleurs traducteurs et metteurs en scène tchèques de l'époque, de même que les rôles dans les pièces ont été attribués aux acteurs les plus doués du Théâtre National. 
giralducien a provoqué le grand intérêt du public tchèque assez habitué jusqu'alors aux pièces françaises des genres comique ou de boulevard. Ce qui a eu comme conséquence d'un côté la déception de cette partie du public - partisans de morceaux frivoles, plus gais et moins ironiques et spirituels, et de l'autre côté enchantement du public plus instruit qui dans la personne de Giraudoux reconnaissait un grand auteur aux valeurs classiques. Néanmoins tout spectateur tchèque était toujours prêt à décrypter les idées cachées dans les œuvres d'art et ainsi à réfléchir sur la symbolique giralducienne cachée dans son théâtre. Dans l'Europe de la deuxième moitié des années trente, où grondait le menace d'un conflit armé, les gens étaient devenus plus sensibles à la voix de Giraudoux et à refléchissaient aux thèmes de la guerre et de la paix, de la responsabilité individuelle ainsi que de la conscience collective. Même si le côté purement français, intellectuel et aussi poétique des pièces giralduciennes a d'abord posé problème au public tchèque, les spectateurs tchèques ont appris à y voir le reflet de leurs difficultés d'alors. D'où le grand succès de Siegfried, de La guerre de Troie n'aura pas lieu et d'Electre. Or, en dernier ressort, le succès du théâtre giralducien en Tchécoslovaquie dans la période de l'entre-deux-guerres est dû autant aux valeurs dramatiques qu'aux questions prédominantes qui habitaient la société de l'époque.

\section{BIBLIOGRAPHIE}

B. (26/4/1929), Siegfried de Giraudoux au Théâtre National, Lidové noviny, Praha, p. 7.

BÁR Zdeněk [-zb-] (1929/1930), La scène morave d'Ostrava, Rozpravy Aventina, tome 5, p. 119.

BRousIL Antonín Matěj (13/4/1938), Electre conversationnelle de Giraudoux, Venkov, Prague, année 33, p. 6.

FISCHER Otokar [Ot.F.] (17/3/1931), Jean Giraudoux : Nový Amfitryon, Lidové noviny, Prague, p. 7.

Fư̌í́ Julius [-jef-] (13/4/1938), La Nouvelle Electre, Rudé právo, Prague, sans pagination.

Götz František [G.] (1933/1934), Le poème dramatique de Giraudoux, Národní divadlo, tome 11 , № 8 , p. 3 .

JELÍNEK Hanuš (1928/1929), Théâtre, Lumír, Prague, tome 55, № 9, pp. 429-431.

JELÍNEK Hanuš (1933/1934), Théâtre, Lumír, Prague, année 60, № 3, p. 172.

JELÍNEK Hanuš (1936/1937), Répertoir, Lumír, Prague, année 63, № 2, p. 119.

JELÍNEK Hanuš (1937/1938), Répertoir, Lumír, Prague, année 64, № 6, p. 326.

-JP- (7/12/1928), Siegfried, České divadlo, Prague, p. 7, 132.

PAULÍK Jaroslav Jan (1928/1929), Jean Giraudoux : Siegfried, Rozpravy Aventina, Prague, tome 4, p. 332.

PETR Jaroslav [Pt] (29/10/1936), Théâtres, Československé divadlo, tome XIX, p. 166.

PíšA A. M. [AMP.] (17/3/1931), 38. Amfitryon, Právo lidu, Prague, № 65, page illisible.

PÍŠA A. M. [AMP.] (13/4/1938), Le mythe antique, Právo lidu, Prague, p. 4. 
ŠRÁMEK Jiř́ (2006), Cyrano de Bergerac d'Edmond Rostand sur les scènes moraves, Studia romanistica 6, Ostrava, p. 194.

TRÄGER Josef [-äg-] (11/1/1934), Aventure du rêve, Právo lidu, Prague, page illisible.

WEINER Richard [R.W.] (16/5/1928), Des théâtres parisiens, Lidové noviny, Praha, p. 7.

WEINER Richard [R.W.] (23/11/1929), Des théâtres parisiens, Lidové noviny, Praha, p. 9.

WINTER Gustav [-ter-] (21/12/1935), Deux grandes pièces, Právo lidu, Prague, p. 6.

\begin{abstract}
The paper deals with the reception of plays Siegfried, Amphitryon 38, Intermezzo and La guerre de Troie n'aura pas lieu, written by the French author Jean Giraudoux, in Czechoslovakia in the period between the two wars. Both the public and the reviewers paid close attention to Giraudoux's plays staged in the period of the First Republic. Each author's play of this period was staged within one year at the latest by the National Theatre in Prague and, in two cases, also by the Regional Theatre in Brno and the Moravian-Silesian Theatre in Ostrava. The critical responses collected from the contemporary press confirm the efforts of the Czechoslovak cultural public to discover the author's intellectual message and to interpret it topically, with respect to the Czech context on the European scale.
\end{abstract}

